\title{
Philosophiques
}

\section{Définition d'orientations théoriques et construction de procédures en analyse du discours}

\section{Jean-Jacques Courtine}

Volume 9, numéro 2, octobre 1982

URI : https://id.erudit.org/iderudit/203194ar

DOI : https://doi.org/10.7202/203194ar

Aller au sommaire du numéro

Éditeur(s)

Société de philosophie du Québec

ISSN

0316-2923 (imprimé)

1492-1391 (numérique)

Découvrir la revue

Citer cet article

Courtine, J.-J. (1982). Définition d'orientations théoriques et construction de procédures en analyse du discours. Philosophiques, 9(2), 239-264.

https://doi.org/10.7202/203194ar
Résumé de l'article

Ce texte répond à trois objectifs : 1) dégager quelques éléments théoriques et méthodologiques essentiels au travail en Analyse du Discours, dans une perspective qui situe le discours comme objet dans un rapport déterminé entre langue et idéologie; 2) exposer la mise en oeuvre de ces éléments à travers la démarche suivie dans un travail d'Analyse du Discours ayant eu pour objet un corpus de discours du Parti communiste français adressés aux Chrétiens (de 1936 à 1976); 3) indiquer le type de résultats auxquels de telles orientations peuvent conduire : une redéfinition des notions de corpus, d'énoncé et d'énonciation dans l'analyse linguistique des processus discursifs. 


\title{
DÉFINITION D’ORIENTATIONS THÉORIQUES ET CONSTRUCTION DE PROCÉDURES EN ANALYSE DU DISCOURS
}

\author{
par Jean-Jacques Courtine
}

RÉSUMÉ. Ce texte répond à trois objectifs: 1) dégager quelques éléments théoriques et méthodologiques essentiels au travail en Analyse du Discours, dans une perspective qui situe le discours comme objet dans un rapport déterminé entre langue et idéologie 2) exposer la mise en oeuvre de ces éléments à travers la démarche suivie dans un travail d'Analyse du Discours ayant eu pour objet un corpus de discours du Parti communiste français adressés aux Chrétiens (de 1936 à 1976); 3) indiquer le type de résultats auxquels de telles orientations peuvent conduire: une redéfinition des notions de corpus, d'énoncé et d'énonciation dans l'analyse linguistique des processus discursifs.

\begin{abstract}
ABSTR ACT. This paper aims at three goals: 1) to draw some essential theoretical and methodological elements from the work's in the field of Discourse Analysis, in a perspective that situates discourse as a certain relation between language and ideology; 2) to expose the use of those elements through the approach followed in a Discourse Analysis research which took a corpus of speeches of the French Communist Party addressed to the Christian Community (1936-1976) as its object; 3) to indicate the type of results to which such orientations may lead: a redefinition of the notions of corpus, enounced and enounciation in the analysis of discourse processes.
\end{abstract}

Ce texte répond à deux objectifs: (1) dégager quelques éléments théoriques et méthodologiques essentiels à un ensemble de travaux d'analyse du discours (notée dorénavant: $A D$ ) récemment effectués (COURTINE, . 1979; 1981; COURTINE et LECOMTE, 1980; COURTINE et MARANDIN, 1982); (2) rendre compte de la mise en oeuvre de ces éléments à travers le projet 
et la démarche suivis dans un travail d'AD ayant pour objet un corpus de discours politique (COURTINE, 1981), en indiquant le type de résultats auxquels de telles orientations peuvent conduire.

\section{REMARQUES INTRODUCTIVES}

1.1 Soulignons tout d'abord les thèses qui articulent la conception que nous présentons ici.

(1) Il existe un ordre du discours, que nous désignons comme matérialité du discursif, distinct de l'ordre de la langue.

(2) La matérialité du discursif consiste en un rapport déterminé entre la langue et l'idéologie.

Nous préciserons ces deux propositions - dans l'esprit des formulations de M. PECHEUX (1975) - en avançant que le discursif matérialise le contact entre l'idéologique et le linguistique, au sens où il représente à l'intérieur de la langue les effets des contradictions idéologiques et où, inversement, il manifeste l'existence de la matérialité linguistique à l'intérieur de l'idéologie. Cela entraîne de notre point de vue les conséquences suivantes:

a) Le discours comme objet doit être pensé dans sa spécificité. L'adoption d'une conception spécifiquement discursive doit éviter, s'il est vrai que le discours peut être pensé comme un rapport entre le linguistique et l'idéologique, de réduire le discours à l'analyse de la langue, ou de le dissoudre dans le travail historique sur l'idéologie comme «représentation». Il s'agit plutôt de tenir à la fois l'analyse linguistique, dont certaines procédures - notamment syntaxiques - fournissent la langue de description et la technique de manipulation des séquences discursives, et l'analyse historique des conditions de formation des ensembles idéologiques comme discours. Et cela en prenant en compte la matérialité discursive comme objet propre, c'est-à-dire en produisant à son endroit des propositions théoriques.

b) Ces propositions théoriques doivent conduire à l'établissement de procédures qui viennent en réaliser le montage 
instrumental, sous la forme d'un dispositif dans un champ méthodologique. La matérialisation en procédures déterminées d'un corps de propositions théoriques visant le discours comme objet de connaissance donne prise sur le discours comme objet empirique-concret, ou objet réel. C'est la condition à laquelle les expressions «l'objet de l'analyse du discours», ou encore «le discours comme objet» peuvent être employées.

Ajoutons que la construction de tels dispositifs est aussi une condition d'explication des propositions théoriques, dans la mesure où un montage instrumental donne de ces propositions une représentation transformée qui les «fait voir». C'est enfin une condition de reproductibilité et de falsifiabilité d'une problématique.

\subsection{L'AD travaille ainsi un objet inscrit dans le rapport de la langue} à l'bistoire. Nous voudrions à cet égard souligner ce qui nous parait, dans les travaux d'AD qui se donnent un tel rapport, constituer un acquis théorique important: il s'agit du concept de formation discursive d'une part, de la distinction entre processus discursifs et base linguistique d'autre part.

Si le processus discursifs constituent la source de la production des effets de sens dans le discours, la langue, pensée comme une instance relativement autonome, est le lieu matériel où se réalisent les effets de sens. Ce que P. HENRY (1975: 94) a pu formuler ainsi:

La notion d'autonomie relative de la langue caractérise l'indépendance d'un niveau de fonctionnement du discours par rapport aux formations idéologiques ${ }^{1}$ qui s'y trouvent articulées, niveau de formation relativement autonome dont la linguistique fait la théorie (...) En d'autres termes, nous posons que tout discours "concret» est doublement déterminé, d'une part par des formations idéologiques qui rapportent ce discours à des formations

1. «On parlera de formation idéologique pour caractériser un élément susceptible d'intervenir comme une force confrontée à d'autres forces dans la conjoncture idéologique caractéristique d'une formation sociale à un moment donné; chaque formation idéologique constitue ainsi un ensemble complexe d'attitudes et de représentations qui ne sont ni individuelles, ni universelles, mais se rapportent plus ou moins directement à des positions de classes en conflit les unes par rapport aux autres» (PÊCHEUX et coll, 1971: 102). 
discursives ${ }^{2}$ définies, d'autres part par l'autonomie relative de la langue, mais nous posons qu'il n'est pas possible de tracer a priori une ligne de démarcation entre ce qui relève de l'une ou l'autre de ces déterminations.

La distinction entre base linguistique et processus discursifs/idéologiques se développant sur cette base découle des thèses posées plus haut, en ce qu'elle fait du rapport du linguistique à l'idéologique, la matérialité même du discursif: elle peut autoriser ainsi la prise en compte des rapports d'antagonisme, alliance, recouvrement, absorption... entre des formations discursives relevant de formations idéologiques déterminées, et exprimer ainsi le fait que dans une conjoncture donnée de l'histoire d'une formation sociale, caractérisée par un certain état des rapports sociaux, des sujets parlants, pris dans l'histoire, puissent s'accorder ou s'affronter sur le sens à donner aux mots, parler différemment, tout en parlant la même langue.

Une mise au point s'impose ici. La catégorisation de l'instance idéologique (formations idéologiques, fonctions discursives...) qui nous permet ici de représenter «l'extérieur de la langue» s'inscrit dans la perspective du travail althussérien à propos des idéologies. Le développement de cette position en $\mathrm{AD}$ a pu prendre la forme du projet volontariste d'une «théorie du discours» (PECHEUX, 1975) duquel nous voulons démarquer notre travail.

La référence à cette expression renvoie en effet, dans le champ de l'AD, à une position théoriciste qui a consisté à mettre en avant "l'articulation théorique» de trois «régions de connaissance»: le marxisme, une théorie freudo-lacanienne du sujet, la linguistique enfin; bref, une version "de gauche» de l'ectoplasme interdisciplinaire qui hante les sciences humaines et sociales.

Ill convient ici de rappeler qu'une interdisciplinarité organique ne saurait se constituer par la juxtaposition de discipli-

2. Les formations ịdéologiques comportent, comme une de leurs composantes, «une ou plusieurs formations discursives inter-reliées qui déterminent ce qui peut et doit être dit (articulé sous la forme d'une harangue, d'un sermon, d'un pamphlet, d'un exposé, d'un programme, etc....), à partir d'une position donnée dans une conjoncturé... (PÊCHEUX et FUCHS, 1975: 11). Nous revenons plus loin sur.le concept de formation discursive. 
nes supposées renfermer a priori des éléments de rigueur scientifique de nature à éclairer un problème déterminé à propos duquel elles détiendraient chacune des "points de vue» certes différents, mais très probablement complémentaires, et ceci pour la bonne raison que, dans le cas qui nous occupe, le discours ne constitue en rien un objet pour les trois «régions de connaissance» en question. Bien au contraire, c'est le travail théorico-pratique du discours comme objet (c'est-à-dire le travail de la contradiction entre objet réel et objet de connaissance) qui fait surgir la référence à l'articulation interdisciplinaire en lui assignant un contenu et une configuration précise. Bref, la position théoriciste a consisté à substituer au travail nécessaire d'une contradiction l'énoncé de sa résolution théorique, c'est-à-dire à supposer le problème théoriquement résolu avant même de l'avoir pratiquement posé.

Ces remarques posent la question de l'intervention du marxisme dans le champ de l'AD; si cette dernière tente d'appréhender des objets historiques que traverse la lutte de classes, si tout discours concret matérialise une position déterminée dans la lutte idéologique, il est alors possible de soutenir que le sens de la référence au marxisme dans son champ soit de venir y rappeler le principe du primat de la contradiction sur les contraires ainsi que celui du caractère «négal» de la contradiction (ALTHUSSER, 1975: 148). C'est à partir de ce double principe que doit se concevoir le recours au marxisme dans notre travail: la contradiction y constitue un élément théorique qui intervient dans la représentation du réel historique, mais aussi un objet d'analyse, au sens où ce sont les modalités discursives d'existence d'une contradiction inégale entre des formations discursives antagonistes qui est l'objet de cette étude.

Ajoutons enfin que nous avons trouvé, dans l'Archéologie du savoir, un écho de la place centrale qu'il convient de faire dans l'ordre du discours, à la question de la contradiction:

Une telle contradiction, loin d'être apparence ou accident du discours, loin d'être cè dont il faut l'affranchir pour qu'il libère enfin sa vérité déployée, constitue la loi même de son existence (...); c'est parce qu'elle est toujours en deçà de lui et qu'il ne peut donc 
jamais la contourner entièrement qu'il change, qu'il sè métamorphose, qu'il échappe lui-même à sa propre continuité. La contradiction fonctionne alors, au fil du discours, comme le principe de son historicité. (FOUCAULT, 1969: 197).

\section{DÉFINITION D'UN PROJET D'ANALYSE DU DISCOURS POLITIQUE}

Ce projet est double: (1) produire l'analyse d'une formation discursive (notée dorénavant: FD) à partir des quelques lignes théoriques que l'on vient d'esquisser; (2) mener une réflexion qui interroge les conditions de possibilité théorique d'une $\mathrm{AD}^{3}$.

2.1 Les descriptions que l'on trouvera plus loin ont été obtenues à partir de l'observation d'un corpus particulier de discours politique: le discours du Parti communiste français, plus précisément le discours communiste adressé aux chrétiens - la "politique de la main tendue» au chrétiens - de 1936 à 1976. Le discours communiste est un objet classique d'étude en $\mathrm{AD}$, mais les travaux qu'il a pu occasionner ne satisfont guère au point de vue développé ici. Comment, en effet, a-t-il été caractérisé?

Soit, et un travail récent de LABBE (1977) vient en donner un exemple, comme un bloc d'immobilité, la clôture d'un espace de répétition, qui prend le sujet parlant dans les mailles de la logique sans faille d'une «grammaire idéologique», bref, un ensemble de discours isolé et clos sur luimême. Soit encore, et ceci dans la tradition d'analyse sociolinguistique du discours (par ex. chez MARCELLESI, 1976), comme un ensemble différentiel, purement «contrastif», s'individuant par proximité ou écart d'autres types de discours, relevant d'une «dialectologie politique» s'attachant à assigner des frontières de classification entre différents types.

Ces tentatives représentent selon nous deux modalités du ratage du discours comme objet, qui conduiraient dans notre perspective à deux interprétations erronées du concept de

3. Nous avons regroupé dans ce texte les éléments théoriques essentiels dé ce projet, ainsi que les types de description et résultats auxquels il peut conduire. Pour une description plus détaillée du corpus pris comme objet, nous renvoyons le lecteur à un texte récent, cité en bibliographie (COURTINE, 1981). 
FD: une FD, ce n'est pas «un seul discours pour tous», ce n'est pas non plus «à chacun son discours», mais ce doit être pensé comme "deux (ou plusieurs) discours en un seul». Deux modalités différentes d'un même oubli: celui de la contradiction comme principe constitutif de toute FD.

Nous considérerons ainsi une FD comme une unité divisée, une hétérogénéité par rapport à soi-même: la clôture d'une FD est fondamentalement instable, elle ne consiste pas en une limite tracée une fois pour toutes séparant un intérieur et un extérieur de son savoir, mais s'inscrit entre diverses FD comme une frontière qui se déplace en fonction des enjeux de la lutte idéologique.

Cela nous conduira plus loin à envisager une redéfinition théorique du concept de FD; on peut cependant d'ores et déjà dégager des éléments développés jusqu'ici ce qui devra être une tâche prioritaire pour l'AD: plutôt que de caractériser isolément ou différentiellement des FD, il faudra repérer les modalités du contact entre FD relevant de formations idéologiques qu'unit et divise en même temps une contradiction inégale; il faudra caractériser les effets discursifs de l'hégémonie idéologique en mettant en évidence les formes selon lesquelles à l'intérieur d'une FD «dominée», des éléments préconstruits produits à l'extérieur d'elle-même sont «intériorisés», c'est-à-dire accueillis, absorbés, reconfigurés, ou au contraire déniés, ou même ignorés...

2.2 Le deuxième volet du projet réside dans le développement d'une réflexion qui interroge les conditions de possibilité d'une $A D$, en proposant un examen critique des notions et procédures qui constituent l'appareillage conceptuel et méthodologique de cette dernière; mais en avançant également, sur chacun des points soumis à l'examen, une redéfinition théorique des éléments critiqués; en construisant enfin des procédures qui rendent opératoires les redéfinitions envisagées et qui garantissent ainsi que ne puisse être critiqué que ce que l'on est en mesure de remplacer.

Cette démarche a porté essentiellement sur les zones suivantes du travail en $A D$ : 
(1) La notion de conditions de production du discours et les opérations de constitution d'un corpus discursif.

(2) La sélection de mots-pôles ou de mots-pivots dans la définition des entrées d'un traitement.

(3) Une conception du rapport énoncé/énonciation en AD.

3. DÉMARCHE SUIVIE DANS L'ANALYSE D'UN PROCESSUS DISCURSIF

\section{1 Éléments critiques}

a) La notion de conditions de production du discours et les opérations de constitution d'un corpus discursif

La notion de conditions de production du discours règle, en $\mathrm{AD}$, le rapport de la matérialité linguistique d'une séquence discursive aux conditions historiques qui déterminent sa production; elle fonde ainsi les procédures de constitution de corpus discursifs (ensemble de séquences discursives dominées par un état donné, suffisamment homogène et stable, des conditions de production du discours).

Cette notion ne connaît cependant bien souvent qu'une définition synchronique où elle tend à se confondre avec ce que les linguistes dénomment «situation d'énonciation». Elle peut être également le lieu d'une confusion entre des déterminations d'ordre psychologique (les représentations subjectives d'une situation de communication attachées à l'aspect "vécu» ou «événementiel» d'un "acte de discours» mettant en présence des locuteurs) et les déterminations proprement historiques qui président à la production comme effets de discours énoncés par des sujets; bref, une inversion imaginaire du réel historique.

Tout cela n'est pas sans conséquence quant à la confection de corpus discursifs: les FD repérées sur cette base sont des ensembles de discours sans mémoire, au sens où le déjà-dit, et notamment les éléments préconstruits (HENRY, 1975; PECHEUX, 1975) dont la production d'une séquence discursive par un sujet énonciateur se soutient, est absent du plan de constitution du corpus 
discursif. Des discours sans mémoire et, nous l'avons souligné plus haut, à l'abri de la contradiction, au sens où une seule FD, - ou au mieux deux FD, conçues comme deux univers parallèles et justaposes de discours - se trouvent représentées dans le corpus.

b) La sélection de mots-pôles et la définition des entrées d'un traitement

Cette procédure consiste à choisir par des moyens divers (hypothèses formulées a priori sur l'importance de tel ou tel vocable dans tel ensemble de discours; considérations d'ordre statistique sur la fréquence de tel item...) un ensemble de termes se comportant comme liste des entrées d'un traitement, à partir de quoi seront effectués divers types de calculs ou de manipulations (indices de récurrence de telle forme; analyses de cooccurrences; constitution de classes d'équivalence distributionnelles...).

Les comptages de mots relèvent d'une optique présyntaxique qui ignore le fonctionnement du discours comme matérialité linguistique et ne peut qu'aboutir à une démographie discursive qui sera au mieux indicative. Les choix effectués a priori sont l'endroit d'un recouvrement non contrôlé entre les «jugements de savoir de l'analyste» (GUILHAUMOU et MALDIDIER, 79) sur l'importance à conférer à tel élément-pivot et la position effective, syntaxiquement descriptible, de cet élément dans le «domaine de savoir» ${ }^{4}$ d'une FD.

Donnons, en anticipant quelque peu par rapport à l'exposition de procédures que nous proposerons plus loin, la solution que nous avons adoptée: puisqu'il s'agit de repérer des «thèmes de discours", nous avons choisi de donner une base formelle - c'est-à-dire ici syntaxique - à de telles procédures de repérage, et cela à partir de certaines structures syntaxiques à propos desquelles nous faisons l'hypothèse qu'elles ont pour effet d'assigner à un élément lexical déterminé l'interprétation: «thème de

4. Ce terme est défini plus loin (au \#3.2 a). 
discours». C'est le sens du recours que nous faisons aux structures de phrase clivées, pseudo-clivées et pseudoclivées inversées, du type:

$$
\left\{\begin{array}{l}
\text { C'EST X QU P } \\
\text { CE QU P C'EST X } \\
\text { X C'EST CE QU P }
\end{array}\right\}
$$

en tant qu'elles constituent une base formelle de localisation et d'identification d'un élément $\mathrm{X}$ du discours.

\section{c) Le traitement du rapport énoncélénonciation en $A D$}

La notion d'énoncé n'a pas de statut théorique en $\mathrm{AD}$, où elle désigne fréquemment la réalisation d'une phrase en surface, ou encore une succession de phrases («énoncé suivi») dont les lois d'enchaînement ont été jusqu'ici peu étudiées.

L'énoncé figure parfois comme proposition logique, «atomes» de discours de la tradition logiciste, dont les combinaisons produisent le texte. Il arrive également qu'on lui adjoigne un supplément pragmatique destiné à en faire un «acte de discours».

Il nous paraît urgent de sortir l'énoncé de la domination d'une problématique de la langue, ou de la logique, et d'en donner une conception spécifiquement discursive, comme FOUCAULT (1969: 111) nous y invite en nous rappelant que l'énoncé ce n'est ni une phrase, ni une proposition logique... ni d'ailleurs, ajoute-t-il, un acte de langage ${ }^{5}$.

Dans la tradition d'une «linguistique de la parole», l'énoncé s'inscrit par ailleurs dans une opposition bipolaire avec l'énonciation. La reprise de cette dernière notion

5. En ce qui concerne la redéfinition du rapport énoncé/énonciation que nous proposerons plus loin (cf. 3.2 a) et b)), nous voulons souligner que c'est l'un des points où la relecture de l'Archéologie du savoir nous a paru particulièrement féconde. FOUCAULT pose le rapport ainsi: on peut parler du même énoncé, là ou il y a plusieurs énonciations distinctes. "L'énonciation est un événement qui ne sè répète pas. Elle a une singularité située et datée qu'on ne peut pas réduire» (FOUCAULT, 1969: 134́). L'énoncé, en revanche, est lié à la notion de répétition. Si l'on neutralise l'énonciation, son temps et son lieu, le sujet qui l'accomplit et les opérations que le sujet utilise, "ce qui se détache, est une forme qui est indéfiniment répétable et peut donner lieu aux énonciations les plus dispersées». (ibid.). 
en $\mathrm{AD}$ a placé le sujet du discours dans un modèle de production des séquences discursives à partir de phrases de base par le biais de règles grammaticales (transformations) qui se surchargent, avec le statut d'«opérations d'énonciation», d'un contenu psychologique. Couplée à l'assimilation des conditions de production du discours à de simples circonstances «situationnelles», elle contribue à opérer la psychologisation spontanée des déterminations spécifiquement historiques du discours que nous signalions plus haut.

Il convient ainsi, selon nous, de dégager l'énonciation d'une problématique centrée sur le sujet et ses opérations pour tenter de penser, à travers la description des positions de sujet (cette notion est précisée plus loin) dans une $\mathrm{FD}$, le processus de l'assujettissement, pour lequel un individu est constitué en sujet de son discours.

\subsection{Quelques éléments de redéfinition théorique}

Cet ensemble de critiques débouche sur l'élaboration d'un cadre théorique où le problème initialement posé puisse être traité; de ce cadre théorique, nous soulignerons ici deux traits: (1) l'établissement de deux niveaux fondamentaux dans la description d'une FD: celui de l'énoncé et celui de la formulation; (2) le primat assigné à un élément théorique central: l'interdiscours.

a) Le niveau de l'énoncé: description de l'interdiscours d'une FD

Nous avancerons que c'est dans l'interdiscours d'une FD ${ }^{6}$, comme articulation contradictoire de FD et de formations idéologiques, que se constitue le domaine de savoir propre à cette FD. La contradiction est première, constitutive des FD: les objets, ou éléments du savoir, s'y forment.

Le domaine du savoir d'une FD fonctionne comme un principe d'acceptabilité discursive pour un ensemble de formulations (il détermine «ce qui peut et doit être dit»)

6. Le terme d'interdiscours (ainsi que celui d'intradiscours utilisé plus loin) est emprunté à PÊCHEUX (1975) et retravaillé à partir des définitions qu'il en a donné. 
en même temps que comme principe d'exclusion du non-formulable. Il réalise ainsi la clôture d'une FD, frontière dont nous avons plus haut souligné l'instabilité.

L'interdiscours d'une FD doit ainsi être pensé comme un processus de reconfiguration incessante dans lequel le savoir d'une FD est conduit, en fonction des positions idéologiques que cette FD représente dans une conjoncture déterminée, à incorporer des éléments préconstruits produits à l'extérieur de lui-même, à en produire la redéfinition ou le retournement; à susciter également le rappel de ses propres éléments, à en organiser la répétition, mais aussi à en provoquer éventuellement l'effacement, l'oubli ou même la dénégation. L'interdiscours d'une FD, comme instance de formation/répétition/transformation des éléments du savoir de cette FD, peut être saisi comme ce qui règle le déplacement de ses frontières.

Nous appelons énoncé ([E]) les éléments du savoir propre à une FD. Nous concevons l'énoncé comme une forme, ou un schéma général, qui gouverne la «répétabilité» au sein d'un réseau de formulations.

Un réseau de formulations consiste en un ensemble stratifié ou dénivelé de formulations, qui constituent autant de reformulations possibles de [E]. Ce que nous appelons stratification ou dénivellation des formulations renvoie à la dimension verticale (ou interdiscursive) d'un [E] comme réseau de formulations. C'est dans de tels réseaux que se stabilise la référence des éléments du savoir: les objets du discours s'y forment comme préconstruits, les [E] s'y articulent.

C'est également à ce niveau de constitution de l'énoncé comme élément de savoir, sous la domination de l'interdiscours, que doit être située l'instance du sujet universel (ou sujet du savoir propre à une FD, noté: SU), se référant à la place d'où peut s'énoncer: "chacun sait/voit/dit/) comprend que...» pour tout sujet énonciateur venant à énoncer une formulation à partir d'une place inscrite dans la FD. Le savoir propre à une FD ést ainsi formé de l'ensemble des assertions renvoyant à SU et marque bien 
que l'énonçable s'y constitue comme extérieur au sujet qui énonce.

b) Le niveau de la formulation: description de l'intradiscours d'une séquence discursive

Nous noterons [e] une formulation, c'est-à-dire une séquence linguistique (de dimension syntagmatique inférieure, égale ou supérieure à une phrase) qui est une reformulation possible de $[E]$ au sein d'un réseau de formulations et qui vient marquer la présence de $[E]$ dans l'intradiscours d'une séquence discursive dominée par une FD dans laquelle $[E]$ est un élément de savoir.

L'intradiscours d'une séquence discursive apparait ainsi comme le lieu où se réalise la séquentialisation des éléments $d u$ savoir, où la dénivellation interdiscursive des [E] est linéarisée, mise à plat en une surface unique de [e] articulées.

Cette «horizontalisation» de la dimension verticale de constitution de l'énoncé est contemporaine de l'appropriation par un sujet énonciateur (noté: $\mathrm{L}$ ), occupant une place déterminée au sein d'une FD, des éléments de savoir de la FD dans l'énonciation de l'intradiscours d'une séquence discursive, cela dans une situation d'énonciation donnée.

c) En conséquence: discours et effets discursifs

La distinction opérée entre niveau de l'énoncé et niveau de la formulation entraîne certaines conséquences, en ce qui concerne l'usage en $\mathrm{AD}$ des termes de discours et de sujet.

Il nous faut indiquer en effet que ces termes ne dénotent pas des objets donnés a priori, mais plutôt des objets à construire: nous ne nous autoriserons à parler de discours qu'au terme de l'articulation du plan de l'interdiscours et de celui de l'intradiscours; toute caractérisation en termes de fonctionnements ou d'effets discursifs engage ainsi un rapport de l'énoncé à la formulation, de la dimension verticale et stratifiée où s'élabore le savoir d'une FD à la dimension horizontale où les éléments de ce savoir se linéarisent en devenant objets d'énonciation. 
Il en va de même du sujet: s'il n'y a, dans la perspective que nous adoptons, nul «sujet du discours», on repère par contre, au sein d'une FD, différentes positions de sujet qui constituent autant de modalités du rapport du sujet universel au sujet d'énonciation (SU/L), du sujet de l'énoncé au sujet de la formulation.

On appellera domaine de la forme-sujet-à la suite de PECHEUX (1975) - le domaine de description de la production du sujet comme effet dans le discours; cela revient à décrire l'ensemble des différentes positions de sujet dans une FD comme modalités particulières de l'identification du sujet de l'énonciation au sujet du savoir, avec les effets discursifs spécifiques qui y sont liés.

\subsection{Construction de procédures et résultats}

Nous exposerons ici tout d'abord la solution envisagée pour le traitement de la notion de «conditions de production» du discours, puis, plus longuement, celle proposée pour la construction de l'énoncé comme objet.

\section{a) Conditions de production et conditions de formation}

Nous pourrions reformuler, en fonction du cadre théorique qui a été décrit, les critiques adressées à la notion de conditions de production du discours, en indiquant qu'elle opère une confusion des déterminations spécifiques aux deux plans de description dégagés: celui de l'énoncé et celui de la formulation. Il convient donc de la dissocier selon ces deux niveaux et d'en tirer les conséquences quant à la constitution d'un corpus discursif qui matérialise sous la forme d'un montage déterminé les exigences théoriques que requiert le concept de FD.

Il faut d'abord concevoir les déterminations spécifiques au niveau de la formulation: choisir une séquence discursive - en tant qu'elle manifeste la réalisation d'un intradiscours - comme point de référence à partir duquel l'ensemble des éléments du corpus recevra son organisation; rapporter cette séquence discursive à un sujet et à une situation d'énonciation déterminés; montrer comment sujet de l'énonciation et circonstances énonciatives sont 
reférables à des places au sein d'appareils idéologiques dans une conjoncture historique donnée ${ }^{7}$. Nous désignerons l'ensemble d'éléments qui viennent d'être mentionnés comme les conditions de production de la séquence discursive de référence.

On ne saurait cependant en rester là: il faut aussi penser les déterminations spécifiques au niveau de l'énoncé et les articuler aux premières. La mise en séquence des formulations dans l'intradiscours de la séquence discursive de référence s'accomplit en effet sous la dépendance du processus discursif inhérent à la FD qui la domine, de formation discursive de référence (la FD communiste dans le cas présent). Un tel processus discursif est soumis à des conditions spécifiques: c'est sous la dépendance de l'interdiscours que se constitue le savoir propre à une $\mathrm{FD}$, dans les réseaux stratifiés de formulations où se forment les énoncés. Il conviendra ainsi de caractériser les conditions interdiscursives dominant le processus discursif de formation/reproduction/transformation des énoncés au sein de la FD de référence; nous désignerons de telles conditions par le terme de conditions de formation de la $F D$ de référence.

Ces redéfinitions commandent la constitution de corpus discursifs dans la forme desquels est représentable le rapport du discours comme objet à deux éléments théoriques essentiels dans notre perspective: la question de la mémoire bistorique tout d'abord, en tant que le discours politique en est le produit; la nature bétérogène et contradictoire de toute FD en second lieu.

Articuler les conditions de production et les conditions de formation dans un corpus discursif donné, cela consistera en effet à faire correspondre à la séquence discursive de référence un domaine de mémoire, c'est-à-dire un ensemble de séquences discursives qui préexistent à l'énonciation de la séquence discursive de référence au sein d'un processus; c'est à partir du domaine de mémoire que sera caractérisée la formation des énoncés et que seront analysés les

7. C'est le texte de l'Appel aux chrétiens de France, prononcé par G. MARCHAIS à Lyon le 10 juin 1976 , que nous avons retenu comme "séquence discursive de référence». 
effets que produit au sein d'un processus discursif l'énonciation d'une séquence discursive déterminée (effets de rappel, de redéfinition, de transformation; mais aussi effets d'oubli, de rupture, de dénégation du déjà-dit). Ajoutons que le domaine de mémoire consiste en une "pluralité contradictoire» de séquences discursives, dont certaines sont dominées par la FD de référence, alors que dépendent de FD contradictoirement liées à cette dernière par des rapports d'antagonisme, d'étayage, d'alliance, de recouvrement . . etc . ${ }^{8}$.

Ce n'est qu'ainsi, nous semble-t-il, que l'on pourra, à partir des catégories de processus et de contradiction, représenter le rapport entre interdiscours et intradiscours, énoncé et formulation, sujet du savoir d'une FD et sujet énonciateur.

b) La construction de l'énoncé comme objet: l'exemple de l'énoncé divisé

Donnons-nous comme point de départ la présence, dans l'intradiscours, de formulations manifestant des structures syntaxiques de phrase clivée, structures à propos desquelles nous avons fait l'hypothèse qu'elles ont pour effet de mettre un élément $\mathrm{X}$, identifié et localisé, en position de thème de discours. Ainsi:

La violence, ce n'est pas de nous qu'elle vient.

Une telle phrase reçoit généralement une interprétation "contrastive». Il s'agit en effet, dans les termes de GROSS (1977) par exemple, d'une "phrase contrastive à un membre», dérivée de la forme générale:

$$
\mathrm{P}_{1}=\mathrm{XAY} / \mathrm{P}_{2}=\mathrm{X} \mathrm{B} \mathrm{Y} \text {. }
$$

Le contraste prend sa source dans la conjonction de deux phrases $P_{1}$ et $P_{2}$; ces deux phrases ne présentent qu'une différence (A/B), A est alors en contraste avec B. L'un des deux membres de cette forme générale peut être effacé (ce

8. Ainsi, dans le cas qui nous occupe, le domaine de mémoire rassemble les principaux textes du Parti communiste français concernant la politique de la main tendue» de 1936 à 1976, en même temps que les textes antagonistes de la doctrine sociale de l'Église. 
qui est parfois producteur d'ambiguiité): On peut cependant, dans le cas qui nous occupe, reconstruire la partie effacée de la formulation, par un recours au contexte (intra ou interdiscursif). On trouvera en effet, dans les références discursives dominées par la FD communiste:

CE N'EST PAS des communistes (mais aussi des travailleurs, prolétaires, du peuple...) QUE vient la violence, C'EST du capital (mais aussi de la grande bourgeoisie, des monopoles, de l'aristocratie de l'argent . . ) QU'elle vient.

Cette formulation possède les caractéristiques suivantes:

- antagonisme de deux termes (et de leurs substituts synonymiques respectifs) dans le savoir de la FD communiste (communistes, travailleurs, prolétaires vs. capital, bourgeoisie, aristocratie de l'argent...);

- usage contrastif de la copule d'identification (est/n'est pas);

- effacement possible d'un des deux membres de la phrase contrastive;

- application d'une transformation de dislocation + pronominalisation possible sur le membre restant.

L'intradiscours de la séquence discursive de référence nous a servi ce point de départ. Si l'on «balaye» à présent l'interdiscours de la FD communiste - en l'occurrence le domaine de mémoire dans lequel nous avons fait figurer un certain nombre de séquences discursives dominées par la FD catholique - on pourra y retrouver sans peine un ensemble de formulations formant réseau, avec lesquelles la formulation de référence entretient un rapport interdiscursif. Pour en donner la forme générale répétable:

La violence vient des communistes.

On repère ainsi dans l'interdiscours une opposition entre deux éléments:

La violence vient des communistes vs. La violence vient $d u$ grand capital,

manifestant la contradiction entre deux domaines de savoir de FD antagonistes. 
On se trouve en fait ici devant une configuration particulière de paraphrasage discursif ${ }^{9}$, dans laquelle deux formulations, de forme syntaxique déterminée $\left(\mathrm{N}_{1} \mathrm{~V}\right.$ de $\mathrm{N}_{2}$ ), assignent des valeurs semblables (la violence, venir de) à certaines places de cette structure et deux valeurs antagonistes à au moins une place (ici: $\mathrm{N}_{2}$, qui prend deux valeurs antagonistes $\left\{\frac{x}{Y}\right\}$, en l'occurence

$$
\left\{\frac{\text { communistes }}{\text { grand capital. }}\right\}
$$

C'est la présence dans l'interdiscours d'une configuration de paraphrasage discursif de type:

$$
\begin{aligned}
& {[\mathrm{e}]_{1}=\mathrm{P}} \\
& {[\mathrm{e}]_{2}=\mathrm{P}}
\end{aligned}\left\{\frac{\mathrm{x}}{\mathrm{y}}\right\}
$$

où $[e]_{1},[e]_{2}$ représentent deux formulations appartenant à des FD antagonistes;

- P représente un contexte de formulation commun;

$-\left\{\frac{x}{y}\right\}$ deux valeurs antagonistes assignées à une place déterminée du schéma syntaxique de ces formulations;

qui peut déclencher, dans l'intradiscours des séquences discursives dominées par ces $\mathrm{FD}$, une modalité contrastive d'identification syntaxiquement réalisée par une phrase en C'EST X QU P à interprétation contrastive.

Nous voudrions à présent revenir sur les éléments théoriques qui nous ont guidé jusqu'ici pour mettre en évidence, à partir du repérage dans l'intradiscours de formulations en C'EST X QU P à interprétation contrastive et de la construction dans l'interdiscours de la configuration du paraphrasage discursif $P\left\{\frac{x}{Y}\right\}$ qui a été obtenue, un mode particulier de contact entre l'idéologique et le linguistique dans l'ordre du discursif, c'est-à-dire dans l'ordre du rapport de l'énoncé à la formulation.

9. La notion de «paraphrase discursive» recouvre en $\mathrm{AD}$ une procédure qui consiste en la construction de classes d'équivalence distributionnelle, selon la méthode de Z. HARRIS (1952), qui établissent la relation de substitution de n segments discursifs dans un contexte établi comme équivalent. Ces segments seront alors dits "en relation de paraphrasage discursif». Une FD peut ainsi être conçue comme un espace de reformulation-paraphrase. 
Le discursif représente bien à l'intérieur du fonctionnement de la langue les effets de la lutte idéologique:

(*) c'est l'existence de contradictions idéologiques qui dessine dans l'interdiscours des configurations du type $P\left\{\frac{x}{Y}\right\}$ envisagé ci-dessus, dans des conditions formelles de distribution, dans un contexte de formulation $P$ déterminé, des constituants apparaissant en $\{\mathrm{X}, \mathrm{Y}\}$; ceux-ci occupent une position déterminée dans les domaines de savoir des FD, dans l'antagonisme desquelles ces contradictions se matérialisent;

(**) des configurations d'une telle forme coexistent avec la présence dans l'ensemble des séquences discursives appartenant au domaine de mémoire des FD considérées, de formulations qui manifestent une certaine régularité lexicale et syntaxique: les constituants venant occuper les positions $\{\mathrm{X}, \mathrm{Y}, \mathrm{P}\}$ dans $\mathrm{P}\left\{\frac{\mathrm{X}}{\mathrm{Y}}\right\}$ sont récurrents dans ces formulations; on peut également y repérer l'occurrence de formes syntaxiques telles que:

\section{C'EST X QU P MAIS CE N'EST PAS Y QUP C'EST X QU P, MAIS C'EST Y QU $P_{2}$ CE N'EST PAS Y QU P C'EST X QU P}

et d'autres formes syntaxiques de contraste ${ }^{10}$. P $\left\{\frac{\mathrm{x}}{\mathrm{y}}\right\}$ coexiste ainsi avec une zone de «répétabilité» déterminée à l'intérieur des processus discursifs considérés (récurrence de certains éléments lexicaux/de certaines formes syntaxiques).

Le discursif manifeste à l'inverse l'existence de la matérialité linguistique à l'intérieur de l'idéologie:

$\left({ }^{*}\right) \quad$ ainsi l'expression $P\left\{\frac{x}{Y}\right\}$ vient-elle, dans l'interdiscours, matérialiser la frontière des domaines de savoir propres à des FD antagonistes, indiquer, par la

10. Parmi lesquelles on peut noter: les transformations négatives, les relatives déterminatives (qui produisent un effet de clivage contrastif sur leur antécédent), les coordinations de phrase par mais, ou par au contraire, certains usages polémiques des guillemets... etc... 
non-substituabilité des constituants qu'elle met en rapport dans des conditions formelles de fonctionnement de la langue, la ligne de partage entre le formulable et le non-formulable pour chacun des processus discursifs dans l'articulation contradictoire desquels cette frontière se matérialise;

(**) ainsi les formulations où apparaissent les structures syntaxiques qui viennent d'être mentionnées ontelles pour effet, dans l'intradiscours d'une séquence discursive, par l'identification constrastive qu'elles opèrent d'éléments de savoir antagonistes, de pointer, de désigner cette frontière, de l'exbiber comme règle pour tout sujet devant énoncer ou interpréter une telle formulation.

Une expression telle que

$$
\text { La violence vient } \quad\left\{\frac{\text { des communistes }}{\text { du grand capital }}\right\}
$$

constitue donc un énoncé. Nous appelons cet énoncé: énoncé divisé et lui attribuons la forme générale $[\mathrm{E}]=\mathrm{P}$ $\left\{\frac{\mathrm{x}}{\mathrm{y}}\right\}$. Nous énumérons ci-dessous un certain nombre de caractéristiques et de conditions attenantes à cette forme.

(1) $\{\mathrm{X}, \mathrm{Y}\}$ y représentent despositions référentielles, dans le contexte de formulation $P$, occupées, à l'intérieur d'un processus discursif donné (et/ou dans deux - ou éventuellement plusieurs processus discursifs), par deux ensembles d'éléments prenant une valeur antagoniste à l'intérieur du processus discursif (et/ou dans deux ou plusieurs - processus discursifs contradictoirement liés).

Nous appelons ces ensembles d'éléments classes référentielles.

$$
\text { Soit } \begin{aligned}
& X=\{a, b, c, d, \ldots\} \\
& Y=\{f, g, h, i, \ldots\} \\
& \text { telles que l'on ait }\left\{\frac{\mathrm{a}}{\mathrm{f}}\right\}\left\{\frac{\mathrm{b}}{\mathrm{g}}\right\}\left\{\frac{\mathrm{c}}{\mathrm{h}}\right\} \\
& \left\{\frac{\mathrm{d}}{\mathrm{i}}\right\} \ldots .
\end{aligned}
$$


(2) $P\left\{\frac{x}{y}\right\}$ reçoit ainsi l'interprétation «les éléments (morphèmes, syntagmes, formulations) en position référentielles $\{\mathrm{X}, \mathrm{Y}\}$ dans le contexte de formulation $P$ ne sont pas commutables».

(3) Pour que $P\left\{\frac{x}{y}\right\}$ constitue un énoncé, c'està-dire une «forme indéfiniment répétable», pouvant donner lieu à des énonciations dispersées au sein d'un processus discursif, il faut:

$\left.{ }^{*}\right)$ que les ensembles d'éléments qui viennent occuper les positions $\{\mathrm{X}, \mathrm{Y}, \mathrm{P}\}$ soient récurrents et cooccurrents dans un ensemble de formulations à l'intérieur du processus discursif. Si la construction de $\mathrm{P}$ $\left\{\frac{\mathrm{X}}{\mathrm{Y}}\right\}$ a été possible dans l'exemple développé, c'est que l'on pouvait dégager de la récurrence de certains éléments (la violence, venir de, . . .) et de leur cooccurrence dans une structure déterminée, des formes d'invariance qui se répètent dans le processus discursif.

Ainsi $\mathrm{N}_{1} \mathrm{~V}$ de $\mathrm{N}_{2}$

VIOLENCE VENIR DE COMMUNISTES.

Nous appellerons une telle forme d'invariance: base de formulation; les propriétés de "répétabilité» des bases de formulation (récurrence et cooccurrence dans un processus discursif) les désignent comme éléments préconstruits du discours. $\{\mathrm{PX} / \mathrm{PY}\}$ représentent deux bases de formulation, dont la mise en rapport sous une forme déterminée produit un énoncé. On peut donc redéfinir l'énoncé comme forme préconstruite d'articulation d'éléments préconstruits $d u$ discours. 
(**) Que $\mathrm{P}\left\{\frac{\mathrm{x}}{\mathrm{Y}}\right\}$ soit une condition d'occurrence de formulations de forme C'EST X QU P/CE QU P C'EST X/X C'EST CE QU P et d'autres formes syntaxiques marquant le contraste dans l'intradiscours de séquences discursives dominées par la FD où $P$ $\left\{\frac{X}{Y}\right\}$ est un élément de savoir, de même qu'une condition d'interprétation des effets de sens liés au fonctionnement contrastif de ces formes.

(4) L'existence de $P\left\{\frac{x}{Y}\right\}$ détermine des conditions de reformulation à l'intérieur du processus discursif et $\mathrm{y}$ inscrit une zone de "répétabilité».

Nous appellerons zone de "répétabilité» dans un processus discursif, les formulations ou séquences de formulations où l'on peut repérer les effets de l'existence de $\mathrm{P}\left\{\frac{\mathrm{x}}{\mathrm{Y}}\right\}$, c'est-à-dire où les conditions $\left({ }^{*}\right)$ et $\left({ }^{* *}\right)$ sont remplies.

(5) $P\left\{\frac{x}{Y}\right\}$ s'inscrit dans un rapport déterminé entre interdiscours et intradiscours dans le processus discursif inhérent à une FD; ce rapport fonctionne comme règle pour le processus discursif.

À cette règle on peut donner la forme d'une implication réciproque, qui rend compte de la forme de coexistence des objets figurant au plan de l'interdiscours (énoncés) et de l'intradiscours (formulations), soit

\section{$P\left\{\frac{X}{Y}\right\} \longleftrightarrow C^{\prime} E S T X$ QU P/CE N'EST} PAS Y QU P.

(6) Cette règle (au sens de régularité existant dans le corpus) opère, pour la zone de «répétabilité» qui nous intéresse ici, le partage entre le formulable et le non-formulable, fixe les limites de l' "acceptabilité discursive», assurant ainsi la clôture d'un domaine de savoir. 
Nous conclurons cet ensemble de développements sur deux points: la question du sujet tout d'abord et un retour sur la définition de l'énoncé, précisant ce qu'il ne saurait être en AD.

\section{Pour conclure: à propos du sujet et de l'énoncé}

4. 1 La définition d'une position de sujet. La zone de fonctionnement discursif liée à l'énoncé divisé se caractérise par le fait que les effets discursifs qu'on y repère entrent dans le registre du fonctionnement polémique du discours: la reformulation par un sujet énonciateur dans l'intradiscours d'une forme d'énoncé $P\left\{\frac{x}{Y}\right\}$ se réalise à travers les formes linguistiques de la réfutation.

On peut ainsi tirer, de la description de l'énoncé divisé qui vient d'être faite, les conséquences suivantes quant à la question du sujet: $P\left\{\frac{\mathrm{x}}{\mathrm{Y}}\right\}$ représente bien, en tant qu'élément de savoir, une expression renvoyant au sujet universel de la FD; l'énoncé vient donner une forme déterminée à ce que nous avons désigné comme l'extériorité de l'énonçable pour un sujet.

Cette forme a ceci de particulier, dans le cas de l'énoncé divisé, qu'elle manifeste le rapport entre deux sujets de savoir antagonistes, que l'on pourrait noter, par analogie avec la forme de l'énoncé: $\left\{\frac{\mathrm{SU}_{1}}{\mathrm{Su}_{2}}\right\}$, au sens où $\mathrm{PX}$ est une expression renvoyant à $\mathrm{SU}_{1}$, et $\mathrm{PY}$ une expression renvoyant à $\mathrm{SU}_{2}$.

Si une position de sujet se définit comme un rapport d'identification du sujet énonciateur au sujet universel d'une FD, la spécificité de la position de sujet dans le fonctionnement polémique du discours tient à ce que cette identification, par laquelle un sujet parlant est interpellé/constitué en sujet idéologique, s'effectue en un lieu q'une contradiction divise.

Et si le domaine de la forme-sujet constitue bien le domaine de la description du sujet comme effet dans le discours, à travers les différentes positions de sujet repérables dans une FD, on pourra noter

$$
\left\{\frac{\mathrm{su}_{1}}{\mathrm{su}_{2}}\right\} \longrightarrow \mathrm{L}
$$


La position de sujet «polémique» comme élément de description de la forme sujet dans la FD.

\subsection{L'énoncé en discours}

Quelques remarques, enfin, sur ce que ne saurait être l'énoncé d'un point de vue spécifiquement discursif.

Un schéma général tel que $P\left\{\frac{x}{Y}\right\}$ ne saurait être assimilé à une forme de base de laquelle les structures de surface des phrases en C'EST X QU P à interprétation contrastive pourraient être dérivées; l'interdiscours ne saurait jouer, vis-à-vis de l'intradiscours, le rôle d'une structure profonde (pas plus que d'une "macro-structure textuelle») à partir de quoi on pourrait envisager la génération de l'intradiscours comme «texte». De même, la régularité représentée ci-dessus n'est pas une règle de génération.

Si l'existence de l'énoncé est distincte de celle de la phrase ou du texte, elle l'est tout autant de celle d'une proposition logique. Une forme telle que $P\left\{\frac{x}{Y}\right\}$ n'est pas un axiome, ou un objet abstrait à partir de quoi pourraient être appliquées des règles de déduction. Poser par ailleurs que $\mathrm{X}, \mathrm{Y}$ sont des positions référentielles ne conduit pas à s'interroger sur la valeur de vérité des expressions qui peuvent y figurer.

Ce n'est ni du «sens», ni de la «référence», au sens logique du terme, des objets du discours dont l'AD se préoccupe, mais de leurs formes de coexistence matérielle dans les processus où se constitue le savoir des FD.

La suite de dénégations qui vient d'être formulée désigne cependant l'existence de l'énoncé comme un lieu problématique de notre démarche. Ces dénégations ont leur raison dans notre intention de donner un statut spécifique à des objets (FD, énoncé, formulations ...) qui les «fasse exister» en dehors des représentations linguistiques ou logiques dans lesquelles ils se coulent spontanément. Voie tâtonnante, entachée d'empirisme, qui emprunte ça et là, condamnée à ne manipuler que des objets concrets-abstraits, produits de généralisations à partir d'observations empiriques. 
L'analyse du discours nous semble actuellement vouée à suivre ce chemin malaisé si elle désire constituer le discours comme objet, en dehors de son rabattement sur une problématique de la langue ou de la logique.

Groupe de recherches en analyse du discours (GRAD)*

UER, d'Informatique et Mathématiques

en Sciences Sociales

Université de Grenoble II

* Le GRAD est une équipe de recherche de l'UER «Informatique et Mathématiques en Sciences Sociales» de l'Université de Grenoble II, qui se consacre à des recherches linguistiques et informatiques prenant le discours comme objet. 


\section{BIBLIOGRAPHIE}

ALTHUSSER, L. (1975). Positions. Hachette, Paris.

COURTINE, J.J. (1979). "Mémoire et discours», à paraître dans Texte et institution. Hurtubise, Montréal, 1982.

(1981). «Quelques problèmes théoriques et méthodologiques en analyse du discours, à propos du discours communiste adressé aux chrétiens», dans Langages, no 62, Didier/Larousse, Paris, pp. 9-128.

COURTINE, J.J. et LECOMTE, A. (1980). «Formation discursive et énonciation», dans Théorie et pratique de la sociolinguistique. Université de Rouen.

COURTINE, J.J. et MARANDIN, J.M. (1982). «Quel objet pour l'analyse du discours?» dans Matérialités Discursives. Presses Universitaires de Lille.

FOUCAULT, M. (1969). L'archéologie du savoir. NRF, Gallimard, Paris.

GUILHAUMOU, J. et MALDIDIER, D. (1979). "Courte critique pour une longue histoire», dans Dialectiques, no 26. Paris.

GROSS, M. (1977). «Une analyse non présuppositionnelle de l'effet contrastif: l'extraction dans c'est . . . que et la négation», dans Linguisticae Investigationes, I: 1, J. Benjamins, Amsterdam.

HARRIS, Z.S. (1952). "Discourse analysis», dans Language, vol. 28, pp. 1-30; trad. française dans Langages, no 13, mars 1969, Didier/Larousse, Paris.

HENRY, P. (1975). «Constructions relatives et articulations discursives» dans Langages, no 37, Didier/Larousse, Paris.

LABBE, D. (1977). Le discours communiste. Presses de la Fondation Nationale des sciences politiques, Paris.

MARCELLESI, J.B. (1976). "Analyse de discours à entrée lexicale», dans Langages, no 41, Didier/Larousse, Paris.

PÊCHEUX, M. (1975). Les vérités de la Palice. Maspéro, Paris.

PÊCHEUX, M., HAROCHE, C. et HENRY, P. (1971). «La sémantique et la coupure saussurienne: langue, langage, discours» dans Langages, no 24, Didier/Larousse, Paris, pp. 93-106.

PÊCHEUX, M. et FUCHS, C. (1975). «Mises au point et perspectives à propos de l'AAD" dans Langages, no 37. Didier/Larousse, Paris, pp. 7-80. 